\title{
Sharp extremal bounds on information diffusion capacities of mobile sensor networks
}

\author{
Jesse Geneson
}

geneson@gmail.com

\begin{abstract}
In this paper, we prove that the maximum possible information diffusion capacity of a mobile sensor network with $n$ agents is equal to $1-\frac{n-2}{n^{2}}$, and the minimum possible capacity is equal to $\frac{2 n+2}{3 n}$, assuming that each pair of agents communicates exactly once. Our upper bound shows that the collector-distributor construction of (Gu et al, 2018) attains the maximum possible capacity of any mobile sensor network where agents are limited to communicating exactly once.

For mobile sensor networks where agents can communicate any number of times and there are a total of $m$ communications, we prove that the maximum possible capacity is equal to $1-\frac{\left(\begin{array}{c}n-1 \\ 2\end{array}\right)}{m n}$ for $m \geq n$ and we also prove that the minimum possible capacity is equal to $\frac{2}{n}$.

We also discuss an error in the proof from (Gu et al, 2018) that the capacity in the random combinatorial model approaches 1 with high probability as the size of the network increases. However, we show that their gossip algorithm proof technique works for a less restricted version of the model.
\end{abstract}

\section{Introduction}

Mobile sensor networks are sensor networks in which the sensors are able to move. These networks can be used for many applications where standard sensor networks would not suffice, such as animal and vehicle monitoring. By improving surveillance quality, mobility can also compensate for having fewer sensors in a network [2].

In addition, mobile sensor networks can reduce computational cost for video surveillance systems [6] and can be used to monitor unfriendly environiments that would be unsuitable for humans or large robots [7]. Such 
networks have also been used in pursuer-evader games and for an implementation of data ferries $[4,5,6]$.

$\mathrm{Gu}$ et al [1] examined the question of how efficiently can a mobile sensor network diffuse information. They focused on several different kinds of mobile sensor networks, including a combinatorial model, a geometric model, and a realistic model based on GPS data. For each model type, they bounded the capacity of a random mobile sensor network of that type, either with proofs or with experimental evidence.

For the random combinatorial mobile sensor network model, $\mathrm{Gu}$ et al claimed that the capacity approaches 1 with high probability as the size of the network approaches infinity. Their proof of this claim relies on a past result of Pittel [3] about gossip algorithms, but their application of the past result is flawed. We explain later in this paper what is wrong with the proof in $[1]$.

$\mathrm{Gu}$ et al also proved that the capacity of their random geometric model is at most 5/6. In addition, they found experimentally that their random geometric model had the lowest capacity, followed by their realistic model, which was their only other model with capacity under 9/10.

As for extremal bounds, $\mathrm{Gu}$ et al showed that there exist combinatorial mobile sensor networks with capacity at least $1-O(1 / n)$, using a construction with an arrangement of lines that they called the collector-distributor construction. However, they did not determine whether this construction attained the maximum possible capacity.

\section{$1.1 \quad$ Terminology}

The first model that we consider in this paper is the Restricted Combinatorial Mobile Sensor Network (RCMSN) model. This was called the combinatorial setting in [1]. In this model, each pair of agents communicates exactly once. During communication, agents share all of the information packets that they have obtained from past communications. There are a total of $\left(\begin{array}{l}n \\ 2\end{array}\right)$ ! possible orderings for the communication sequence in this model.

We also consider a second model, the Geometric Mobile Sensor Network (GMSN) model (called the geometric setting in [1]). In this model, each agent is restricted to an infinite line, and the movement of agents is coordinated so that communication happens at all intersections. Specifically, agent movement is governed by a vertical line sweeping from left to right.

The third model that we consider is a generalization of the RCMSN model, simply called the Combinatorial Mobile Sensor Network (CMSN) model. In this model, each pair of agents can communicate as many or as 
few times as they would like. The length of the communication sequence is specified by a parameter $m$ in addition to the number of agents $n$.

We represent communication between agents $i$ and $j$ as an information packet $\{i, j\}$, and we say that $\{i, j\}$ can reach node $k$ if some agent shares the information packet $\{i, j\}$ with agent $k$. We call $(\{i, j\}, k)$ a reachable pair if the information packet $\{i, j\}$ can reach agent $k$.

Finally, we can define information diffusion capacity for each network type. Define $S$ to be the total number of reachable pairs. For an RCMSN

or a GMSN, the capacity is defined as $\frac{S}{n\left(\begin{array}{c}n \\ 2\end{array}\right)}$. For a CMSN of length $m$ with $n$ agents, the capacity is defined as $\frac{S}{n m}$.

\subsection{New results}

In Section 2, we prove that the maximum possible capacity of an RCMSN or a GMSN with $n$ agents is equal to $1-\frac{n-2}{n^{2}}$. We also prove that the minimum possible capacity of an RCMSN or a GMSN with $n$ agents is equal to $\frac{2 n+2}{3 n}$.

In Section 3, we prove that the maximum possible capacity of a CMSN of length $m$ with $n$ agents is equal to $1-\frac{\left(\begin{array}{c}n-1 \\ 2\end{array}\right)}{m n}$ for $m \geq n$. We also prove that the minimum possible capacity of a CMSN with $n$ agents is equal to $\frac{2}{n}$.

Finally in Section 4, we discuss the error in the proof from [1] that the capacity in the random RCMSN model approaches 1 with high probability as the size of the network approaches infinity. We show that the proof technique actually works for the random CMSN model, even though it fails for the RCMSN model.

\section{Extremal bounds for RCMSN and GMSN mod- els}

Theorem 2.1. The minimum possible capacity of an RCMSN with $n$ agents is $\frac{2 n+2}{3 n}$.

Proof. Gu et al [1] proved that every RCMSN with $n$ agents has capacity at least $\frac{2 n+2}{3 n}$. Thus it suffices to exhibit an infinite family of combinatorial mobile sensor networks that achieve this lower bound.

Consider the RCMSN with $n$ agents where pairwise communication is ordered lexicographically, so that $\{a, b\}$ precedes $\{c, d\}$ if and only if (1) $a<c$ or (2) $a=c$ and $b<d$. (Note that for this construction, we write pairwise communication between agents $i$ and $j$ as $\{i, j\}$ with $i<j$.) We 
count how many of the $n\left(\begin{array}{l}n \\ 2\end{array}\right)$ pairs of the form $(\{i, j\}, k)$ are reachable in this ordering.

First note that all pairs of the form $(\{i, j\}, i)$ or $(\{i, j\}, j)$ are reachable in this ordering, giving $2\left(\begin{array}{l}n \\ 2\end{array}\right)$ pairs. Moreover for any triple $i<j<k$, both $(\{i, j\}, k)$ and $(\{i, k\}, j)$ are both reachable, giving $2\left(\begin{array}{l}n \\ 3\end{array}\right)$ pairs.

Consider the pair $(\{j, k\}, i)$. In order for this pair to be reachable, $i$ must appear in some pairwise communication that occurs after $\{j, k\}$ in the ordering. However, this is impossible by definition of the ordering.

Thus the capacity for this RCMSN is $\frac{2\left(\begin{array}{l}n \\ 3\end{array}\right)+2\left(\begin{array}{l}n \\ 2\end{array}\right)}{n\left(\begin{array}{c}n \\ 2\end{array}\right)}=\frac{2 n+2}{3 n}$, so the lower bound is achieved.

Corollary 2.2. The minimum possible capacity of a GMSN with $n$ agents is $\frac{2 n+2}{3 n}$.

Proof. The lower bound follows from Theorem 2.2. For the upper bound, define $l_{i}$ as the line through the points $(i, 0)$ and $(0, n+1-i)$ for $1 \leq$ $i \leq n$. The capacity of the arrangement $l_{1}, \ldots, l_{n}$ clearly matches the lower bound.

Theorem 2.3. The maximum possible capacity of an $R C M S N$ with $n$ agents is $1-\frac{n-2}{n^{2}}$.

Proof. For the upper bound, note that for any ordering of pairwise communications, every agent has a last occurrence in the ordering. For all pairwise communications $\{i, j\}$ after an agent $k$ 's last occurrence, the pair $(\{i, j\}, k)$ is not reachable.

Consider any RCMSN with $n$ agents. Let $C$ be the set of pairwise communications that contain two last occurrences of agents in the ordering, let $r=|C|$, and let $S$ be the set of agents in $C$. Note that $n / 2 \geq r \geq 1$ for any ordering. None of the agents see any of the communications in $C$ except for the two agents in each communication, so there are $(n-2) r$ unreachable pairs $(\{i, j\}, k)$ where $\{i, j\}$ is the last occurrence of both $i$ and $j$.

There are $n-2 r$ agents in $A-S$ that have last occurrences in a pairwise communication with another agent that occurs later in the ordering. These agents cannot see any pairwise communications after their last occurrence, so there are at least $\sum_{i=1}^{n-2 r}(i-1)$ unreachable pairs $(\{i, j\}, k)$ where $\{i, j\}$ is the last occurrence of either $i$ or $j$, and $k \in A-S$ has last occurrence before $\{i, j\}$.

Let $c_{1}, \ldots, c_{n-2 r}$ be the pairwise communications (in order) that contain the last occurrence of an agent of $A-S$. Let $b_{i}$ denote the number of communications in $C$ containing agents that never see $c_{i}$, so there are $2 b_{i}$ 
agents in $S$ that never see $c_{i}$, so there are $2 b_{i}$ unreachable pairs $\left(c_{i}, k\right)$ for $k \in S$.

There are $2 r-2 b_{i}$ agents in $S$ that see $c_{i}$ (and $r-b_{i}$ communications in $C$ containing agents that see $c_{i}$ ). For an agent in $S$ to see $c_{i}$, it must either be in $c_{i}$, or it must occur after $c_{i}$ in some pairwise communication with some agent that has already seen $c_{i}$. Thus there must be at least $r-b_{i}-1$ communications containing elements of $S$ that are after $c_{i}$, not in $C$, and not equal to $c_{j}$ for any $j$. So there are at least $r-b_{i}-1$ unreachable pairs $(e, x)$, where $e$ is a communication after $c_{i}$ but not in $C$ such that $e \neq c_{j}$ for all $j$, and $x$ is the agent with last occurrence in $c_{i}$.

Thus for each $i$ with $1 \leq i \leq n-2 r$, there are at least $r-1+b_{i}$ unreachable pairs of the two forms described in the last two paragraphs. Each of these unreachable pairs either has $c_{i}$ and an agent of $S$, or the agent with last occurrence in $c_{i}$ and a communication $e$ after $c_{i}$ but not in $C$ such that $e \neq c_{j}$ for all $j$. In total, every RCMSN with $n$ agents has at least $(n-2) r+$ $(n-2 r)(n-2 r-1) / 2+(n-2 r)(r-1)=n^{2} / 2-3 n / 2+r \geq(n-1)(n-2) / 2$ unreachable pairs, so the capacity is at most $\frac{n\left(\begin{array}{c}n \\ 2\end{array}\right)-\left(\begin{array}{c}n-1 \\ 2\end{array}\right)}{n\left(\begin{array}{c}n \\ 2\end{array}\right)}=1-\frac{n-2}{n^{2}}$.

For the lower bound, we could use the collector-distributor construction from [1], but instead we first show an alternative lower bound construction and defer the collector-distributor construction until the following corollary. Consider any communication sequence with $n$ agents with a contiguous subsequence $\left\{a_{1}, a_{2}\right\},\left\{a_{2}, a_{3}\right\}, \ldots,\left\{a_{n-1}, a_{n}\right\},\left\{a_{n}, a_{1}\right\}$, where $a_{1}, \ldots, a_{n}$ is some permutation of $1, \ldots, n$. Note that by the end of the subsequence, $a_{1}$ has received the information packet for every pairwise communication in the whole ordering up to $\left\{a_{n}, a_{1}\right\}$.

For the construction, we assume there are an odd number of agents and that the agents are labeled $0, \ldots, n-1$. We use the shorthand $i+_{n} j$ to denote the remainder when $i+j$ is divided by $n$. Let $m$ be an integer relatively prime to $n$ such that $1<m<n / 2$, e.g. $m=(n-1) / 2$.

For $d$ relatively prime to $n$, let $s_{d}=\left\{0,0+{ }_{n} d\right\},\left\{d, d+{ }_{n} d\right\}, \ldots,\{n-d, 0\}$, which has length $n$. Our lower bound construction is of the form $x s_{m} s_{1}$, where $x$ is any permutation of the pairwise communications not included in $s_{m}$ and $s_{1}$.

Note that by the end of $s_{m}, 0$ has received information on every pairwise communication in the whole ordering up to that point. During $s_{1}$, each agent $i$ passes all of the preceding information to the agent $i+1$ for $0 \leq i \leq n-2$. Thus agent $i$ misses information on $n-i-1$ pairwise communications for $i>0$, and agent 0 has all of the information at the end, which implies that 
the capacity is at least $\frac{n\left(\begin{array}{l}n \\ 2\end{array}\right)-\sum_{i=1}^{n-1}(n-i-1)}{n\left(\begin{array}{c}n \\ 2\end{array}\right)}=1-\frac{n-2}{n^{2}}$.

Corollary 2.4. The maximum possible capacity of a GMSN with $n$ agents is $1-\frac{n-2}{n^{2}}$.

Proof. The upper bound follows from Theorem 2.3. For the lower bound, note that the collector-distributor construction in [1] matches our upper bound. Specifically, for any arrangement of $n-2$ lines in general position, we can add a collector line at the right after all intersections that has the highest slope and intersects all previous lines to the right of their intersections, and then we can add a distributor line which has the most negative slope and intersects all previous lines to the right of their intersections.

\section{Extremal bounds for CMSN Model}

Theorem 3.1. The minimum possible capacity of a CMSN with $n$ agents is $\frac{2}{n}$.

Proof. Any CMSN of length $m$ has at least $2 m$ reachable pairs since every pairwise communication is known to both agents involved. For the upper bound, consider any CMSN where only 2 of the $n$ agents communicate. Alternatively, consider any CMSN where the agents only communicate in pairs.

Theorem 3.2. The maximum possible capacity of a CMSN of length $m$ with $n$ agents is $1-\frac{\left(\begin{array}{c}n-1 \\ 2\end{array}\right)}{m n}$ for $m \geq n$.

Proof. For the upper bound, consider any CMSN with $n$ agents. Let $x$ be the number of agents that appear in the communication sequence. Using the same proof as Theorem 2.3, there are at least $(x-1)(x-2) / 2$ unreachable pairs $(e, y)$ where $y$ is an agent that appears in the communication sequence. There are $(n-x) m \geq(n-x) n$ unreachable pairs $(e, y)$ where $y$ is an agent that does not appear in the communication sequence, giving a total of at least $(n-x) n+(x-1)(x-2) / 2$ unreachable pairs. The function $f(x)=(n-x) n+(x-1)(x-2) / 2$ has partial derivative $\frac{d f}{d x}=x-n-3 / 2$, so the minimum value for $x \in[0, n]$ occurs at $x=n$.

Thus every CMSN with $n$ agents has at least $(n-1)(n-2) / 2$ unreachable pairs, so the capacity is at most $\frac{n m-(n-1)(n-2) / 2}{n m}=1-\frac{\left(\begin{array}{c}n-1 \\ 2\end{array}\right)}{m n}$.

The lower bound construction takes the form $x s_{1}$, where $x$ is any sequence of pairwise communications of length $m-n$ that has agent 0 in each 
pairwise communication, and $s_{1}$ is defined as in Theorem 2.3. As in the proof for RCMSNs, the capacity is at least $\frac{n m-\sum_{i=1}^{n-1}(n-i-1)}{n m}=1-\frac{\left(\begin{array}{c}n-1 \\ 2\end{array}\right)}{m n}$.

\section{Gossip algorithms}

In [1], Gu et al used past results on gossip algorithms [3] to claim that the capacity of a random RCMSN is $1-O\left(\log ^{2}(n) / n\right)$ with high probability. However, the proof in [1] does not work for the RCMSN model. Gu et al also claimed that the capacity of a random CMSN is $1-O\left(n \log ^{2}(n) / m\right)$ with high probability, which is actually true. In this section, we explain why the first claim is false and the second claim is true.

For the first claim, the issue lies in the fact that Pittel's $O(\log (n))$ bound on the number of rounds that it takes for a rumor to diffuse through a gossip network with high probability assumes that the rounds of the random gossip algorithm are independent of each other [3]. Specifically, in the setup for Pittel's result, one of $n$ people knows a rumor. In the first round, they pass the rumor to someone chosen uniformly at random. In each subsequent round, each person that already knows the rumor tells it to someone chosen uniformly at random and independent of all past and present choices.

The erroneous proof from [1] starts with an ordering of the $\left(\begin{array}{l}n \\ 2\end{array}\right)$ pairwise communications in an RCMSN and divides them into groups of size $n \log (n)$. $\mathrm{Gu}$ et al claim that with high probability, every agent occurs in each group. From there, they conclude that each group can be viewed as one round of the gossip algorithm from [3], where every node communicates to some random partner. The issue here is that the random partners are not independent of all past and present choices, since all pairwise communications in an RCMSN are distinct.

Below, we prove Gu et al's claim that the capacity of a random CMSN is $1-O\left(n \log ^{2}(n) / m\right)$ with high probability, since this was stated without proof in [1].

Theorem 4.1. The capacity of a random CMSN of length $m$ with $n$ agents is $1-O\left(n \log ^{2}(n) / m\right)$ with high probability.

Proof. Partition the sequence of $m$ pairwise communications into groups of size $[n \log (n)]+1$ (and one group of possibly smaller size). For any agent $i$, the probability that $i$ does not appear in a given full-size group is at most $\left(1-\frac{n-1}{\left(\begin{array}{c}n \\ 2\end{array}\right)}\right)^{[n \log (n)]+1}<e^{-2 \log (n)}=1 / n^{2}$. Thus the probability that there exists an agent that does not appear in a given full-size group is at most $1 / n$ by the union bound. Therefore the probability that every agent 
appears in every full-size group among the last $O(\log (n))$ groups is at least $1-O(\log (n) / n)$, which approaches 1 as $n \rightarrow \infty$. Thus the probability that every agent appears twice in every consecutive pair of full-size groups among the last $O(\log (n))$ groups approaches 1 as $n \rightarrow \infty$.

Thus we can treat each consecutive pair of full-size groups of pairwise communications among the last $O(\log (n))$ groups as a single round of the gossip algorithm, where every agent has a random partner that is independent of all other past and present partnerings. Indeed, for each consecutive pair of full-size groups, we can assign random partners to agents starting from the first agent, where the next agent assigned a random partner is the random partner $p$ of the previous agent, unless $p$ was already assigned a partner, in which case the next agent assigned a random partner is the lowest-numbered agent.

The probability in a given pair of consecutive full-size groups that $i$ has random partner $j$ is equal for all agents $j \neq i$, regardless of the other random partners in the same group or earlier in the communication process. (Note that this is not true for the random RCMSN model.) By the result of Pittel [3], every pairwise communication before the last $O(\log (n))$ groups can be diffused to every agent with high probability by the end of

the communication process. Thus the capacity is at least $\frac{m n-O\left(n^{2} \log ^{2}(n)\right)}{m n}=$ $1-O\left(n \log ^{2}(n) / m\right)$.

\section{References}

[1] Chen Gu, Ian Downes, Omprakash Gnawali, Leonidas J. Guibas: On the ability of mobile sensor networks to diffuse information. IPSN 2018: $37-47$

[2] Benyuan Liu, Olivier Dousse, Philippe Nain, Donald F. Towsley: Dynamic Coverage of Mobile Sensor Networks. CoRR abs/1101.0376 (2011)

[3] B. Pittel: On spreading a rumor. Journal on Applied Mathematics (1987).

[4] C. Sharp, S. Schaffert, A. Woo, N. Sastry, C. Karlof, S. Sastry, and D. Culler: Design and implementation of a sensor network system for vehicle tracking and autonomous interception. Proc. IEEE European Workshop Sensor Networks, pp. 93-107, 2005. 
[5] A. Somasundara, A. Kansal, D. Jea, D. Estrin, and M. Srivastava: Controllably mobile infrastructure for low energy embedded networks. IEEE Trans. Mobile Computing, vol. 5, no. 8, pp. 958-973, 2006.

[6] You-Chiun Wang, Fang-Jing Wu, Yu-Chee Tseng: Mobility management algorithms and applications for mobile sensor networks. Wireless Communications and Mobile Computing 12(1): 7-21 (2012)

[7] Guangming Song, Yaoxin Zhou, Fei Ding, and Aiguo Song: A Mobile Sensor Network System for Monitoring of Unfriendly Environments. Sensors 2008, 8(11), 7259-7274. 